\title{
A routine electrocardiogram should not be used to determine the size of myocardial infarction in the rat
}

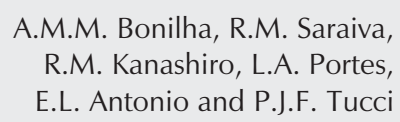

Laboratório de Fisiologia Cardíaca, Universidade Federal de São Paulo, São Paulo, SP, Brasil

\section{Correspondence \\ P.J.F. Tucci \\ Rua Estado de Israel, 181, Apto. 94 \\ 04022-000 São Paulo, SP \\ Brasil \\ E-mail: tucci@fcr.epm.br \\ Research supported by \\ CNPq (No. 300.692/80-3NV) and \\ FAPESP (No. 99/04533-4).}

Received June 18, 2004

Accepted February 10, 2005

\begin{abstract}
Nine lead electrocardiograms of non-infarcted $(\mathrm{N}=61)$ and infarcted ( $\mathrm{N}=71)$ female Wistar rats $(200-250 \mathrm{~g})$ were analyzed in order to distinguish left ventricle myocardial infarction (MI) larger than $40 \%$ (LMI) from MI smaller than 40\% (SMI). MI larger than $40 \%$ clearly caused a deviation of ÂQRS and ÂT from normal values of 270-360 degrees to 90-270 degrees. Infarcted rats showed $Q$ wave in $D_{1}$ larger than $1 \mathrm{~mm}$ with $94 \%$ sensitivity and $100 \%$ specificity. The sum of QRS positivity in $\mathrm{V}_{1}, \mathrm{~V}_{2}$ and $\mathrm{V}_{6}$ lower than $10 \mathrm{~mm}$ identified MI with $82 \%$ sensitivity and $100 \%$ specificity. The data showed that MI can be easily and reliably diagnosed by electrocardiogram in the rat. However, contradicting what is frequently believed, when specificity and sensitivity were analyzed focusing on MI size, none of these current electrocardiographic indices of MI size adequately discriminates LMI from SMI.
\end{abstract}

Key words

- Myocardial infarction size

- Rats

- Electrocardiogram

- Echocardiogram

- ROC curve

\section{Introduction}

Histopathology is the standard method for determining post-mortem myocardial infarction (MI) size in rats. However, it is frequently necessary to define MI size in vivo by a noninvasive method. We have previously demonstrated that MI size evaluated by an echodopplercardiogram (ECHO) shows a good correlation with histopathological measurements (1). However, this is an expensive method that requires special expertise for correct execution. Although in humans the ECG can characterize MI size only with special devices $(2,3)$, it is frequently reported that the nine-lead routine ECG can differentiate left ventricle MI larger than 40\% (LMI) from MI smaller than $40 \%$ (SMI) in the rat (4-9). Our aim was to reevaluate whether the ECG allows identifying LMI in the rat.

\section{Material and Methods}

Female Wistar rats (200-250 g): 37 controls, 24 sham-operated animals and 71 infarcted (MI) animals were cared for in compliance with a protocol approved by the Research Ethics Committee of the Federal University of São Paulo, Brazil.

MI was induced according to a wellaccepted technique (5). Briefly, after ether anesthesia, a left thoracotomy was performed, the heart was exteriorized and the left anterior descending coronary artery ligated with 6-0 polypropylene suture. The heart was 
quickly returned to its position and the thorax immediately closed. In sham-operated animals, the coronary ligation was placed in the same location but not tied tightly.

Five weeks after surgery, ECG and ECHO were performed in animals anesthetized (ip) with a mixture of ketamine $(50 \mathrm{mg} / \mathrm{kg})$ and xylazine $(10 \mathrm{mg} / \mathrm{kg})$. ECG was obtained with a direct recording system. The electrodes were connected to surgical needles and inserted subcutaneously into the four limbs. Records were obtained at $50 \mathrm{~mm} / \mathrm{s}$ paper speed with a sensitivity of $20 \mathrm{~mm} / \mathrm{mV}(2 \mathrm{~N})$ from classical uni- (aVr, aVL, aVF) and bipolar $\left(\mathrm{D}_{1}, \mathrm{D}_{2}, \mathrm{D}_{3}\right)$ limb leads and three $(\mathrm{N}$ $=71)$ or two $(\mathrm{N}=61)$ precordial leads. Precordial leads were placed half-way between the manubrium and the xiphoid process: $V_{1}$ was located to the right of the sternum and $\mathrm{V}_{2}$ (not done when two precordial leads were recorded) to the left of the sternum, and $\mathrm{V}_{6}$ was placed on the left midaxillary line.

Echocardiography was performed by the same person (RMS) using an HP SONOS 5500 instrument (Philips Medical System, Andover, MA, USA) with a 12-MHz transducer at a depth of $2 \mathrm{~cm}$. Chests were shaved, electrocardiographic leads were attached to the limbs and the animals were placed in left lateral decubitus. Two-dimensional and $\mathrm{M}$ mode images from the parasternal longitudinal, transverse and apical views were obtained and recorded on a 0.5 -inch videotape. Transverse images were obtained at three levels: basal (at the tip of the mitral valve leaflets), middle (at the papillary muscle level) and apical (distal to the papillary muscle but beyond the cavity cap). The images were recorded on videotape and imaging analysis and measurements were performed off-line. MI size was estimated as subjective identification of akinesis or dyskinesis. On each echocardiographic transverse plane (basal, middle and apical) the arc corresponding to the segments with MI (AMI) and the total perimeter of the endocardial border (PE) were measured three times at end diastole, and the MI size (MIS) was calculated as: MIS $(\%)=\mathrm{AMI} / \mathrm{PE}$ x 100. The final MIS of each animal was calculated as the mean MIS estimated on the three planes.

Data were analyzed by plotting receiver operating characteristic (ROC) curves (MedCalc Software, version 7.4.0.0., Frank Schoonjans, Belgium) to determine the specificity and sensitivity for each variable.

\section{Results}

Thirty of the infarcted rats (42\%) showed an SMI ranging from 8 to $39 \%$ (mean \pm SD: $26 \pm 10 \%$ ) of left ventricle endocardial circumference and $41(58 \%)$ showed an LMI ranging from 41 to $57 \%(47 \pm 5 \%)$.

The most sensitive and easiest MI size indicator for the rat is the presence of a $\mathrm{Q}$ wave in $\mathrm{D}_{1}\left(\mathrm{Q}_{1}\right)$, which was not observed in non-infarcted rats (100\% specificity), whereas in infarcted animals $\mathrm{Q}_{1}$ showed $94 \%$ sensitivity.

A conspicuous difference could be noted between infarcted and non-infarcted rats regarding ÂQRS (Figure 1A) and ÂT (Figure 1B). There was a similar spatial orientation for ÂQRS in control and sham rats, markedly between $\mathrm{D}_{1}$ ( 0 or 360 degrees) and aVF (270 degrees), whereas in infarcted rats ÂQRS was predominantly located between 90 and 270 degrees, discriminating MI rats with $87.3 \%$ sensitivity and $96.5 \%$ specificity when ÂQRS was less than 270 degrees (Figure 2A). The area under the ROC curve (AUC) for ÂQRS as an indicator of infarcted or non-infarcted rats was 0.919 (Figure 3A). Taking into account that a perfect fit for perfect sensitivity (100\%) and specificity $(100 \%)$ results in a maximal value of 1.0 for the AUC, we can conclude that ÂQRS is a very good indicator of MI in the rat. Similarly, ÂT clearly differentiated infarcted from non-infarcted rats (Figure 1B). As a rule, ÂT was located at about 270 degrees in noninfarcted rats, whereas in MI rats ÂT was 
located between 90 and 270 degrees. The sensitivity of ÂT lower than 240 degrees (Figure 2B) in distinguishing non-infarcted from infarcted rats was $92.5 \%$, specificity was $88.3 \%$ and the AUC was 0.92 .
The sum of QRS positivity $(\Sigma)$ lower than $10 \mathrm{~mm}$ in three precordial leads was restricted to MI rats (100\% specificity) but, unfortunately, not all infarcted rats presented this signal; in fact, $\Sigma$ identified MI with
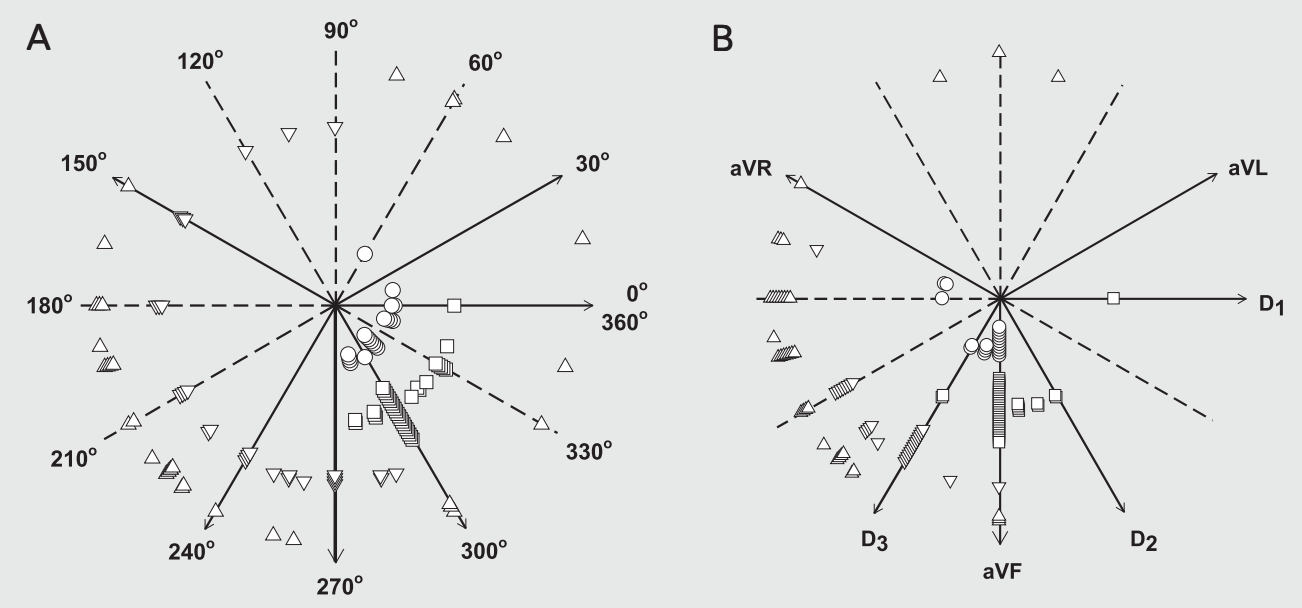

Figure 1. ÂQRS and ÂT for the presence and size of myocardial infarction (MI). Frontal plane localization of ÂQRS (panel A) and $\hat{A} T$ (panel B) in control (squares) and sham-operated (circles) animals and infarcted rats with an $\mathrm{Ml}$ scar occupying more than $40 \%$ of the left ventricular endocardial circumference (triangles) and infarcted rats with an $\mathrm{Ml}$ scar occupying less than $40 \%$ of left ventricular endocardial circumference (inverted triangles).

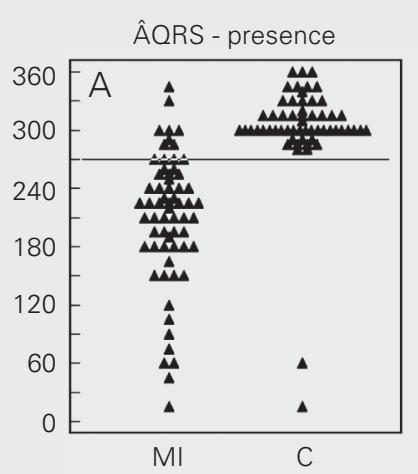

$\leq 270.0$

Sens: 87.3

Spec: 96.5
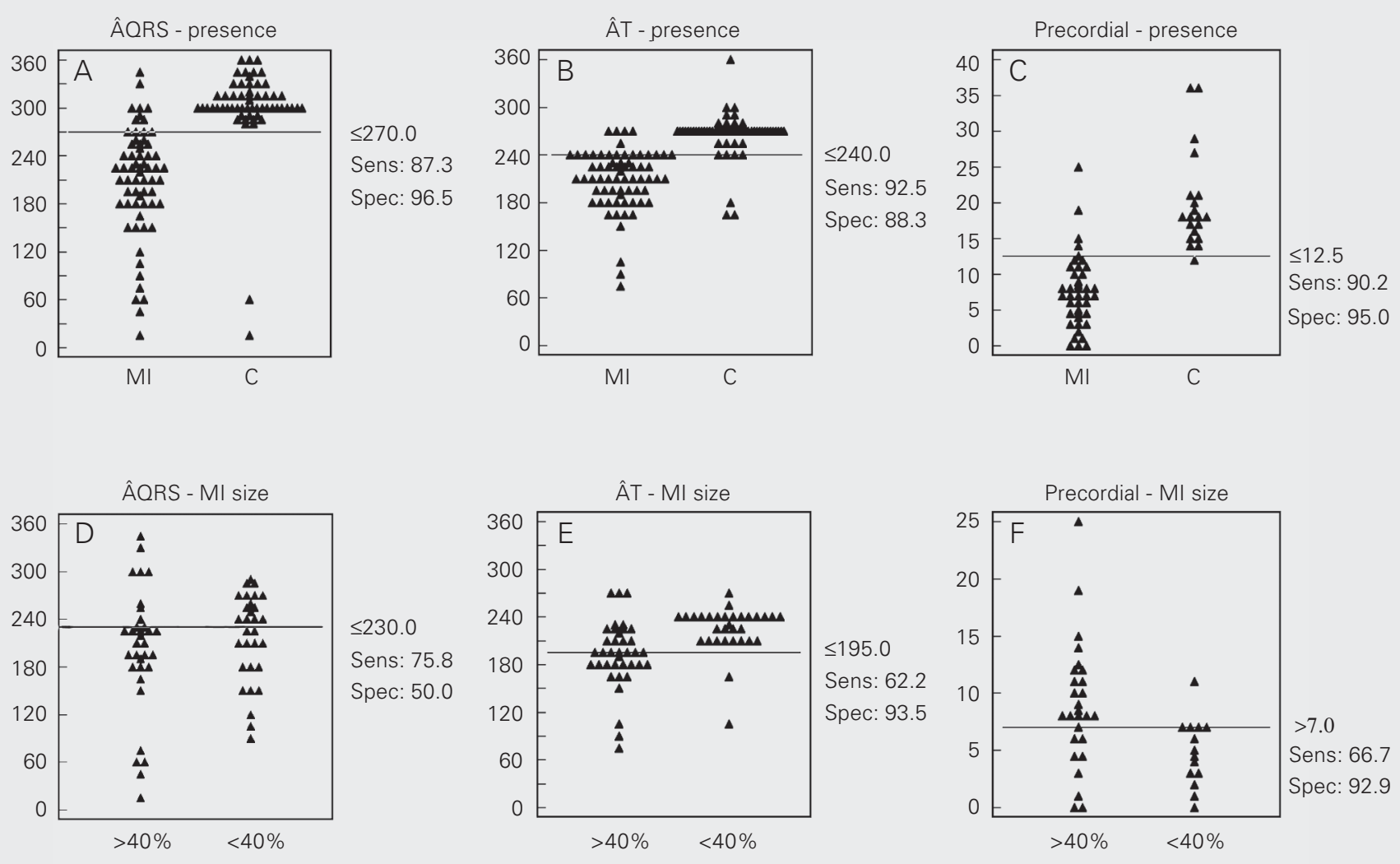

Figure 2. Sensitivity and specificity of ÂQRS, ÂT and precordial R waves. Frontal plane localization of ÂQRS (panels A and D) and ÂT (panels B and E) and the sum of precordial $\mathrm{R}$ waves (panels $\mathrm{C}$ and $\mathrm{F}$ ) arranged according to the presence of myocardial infarction (MI) or absence (C) for rats with an $\mathrm{MI}$ scar occupying more than $40 \%$ (>40\%) and less than $40 \%(<40 \%)$ of the left ventricular endocardial circumference. Sensitivity (Sens) and specificity (Spec) values of the cut-off point for each evaluation (horizontal line) are also shown. 
$75.6 \%$ sensitivity and the AUC was 0.955 (Figure 2C).

In the present study, ECG indicators previously utilized to discriminate LMI from SMI (4-9) were not so effective in this intent as in identifying the presence of MI in the rat. In fact, $Q_{1}>1 \mathrm{~mm}$ was found in $92 \%$ of our rats with LMI, but $96 \%$ of the rats with SMI also showed $\mathrm{Q}_{1}>1 \mathrm{~mm}$. Additionally, no correlation was noted between $\mathrm{Q}_{1}$ depth and MIS. As a result of the poor capacity of ÂQRS to distinguish LMI from SMI, when ÂQRS was judged to discriminate between LMI and SMI, the AUC (Figure 3B) showed values $(0.572)$ very close to the lowest predictable one for this method (0.50). Indeed, it was shown that ÂQRS of less than 230 degrees was the best cut-off point for the differentiation between LMI and SMI (Figure 2D). In this situation, moderate sensitivity $(75.8 \%)$ was associated with very low specificity (0.50). ÂT showed good specificity $(93.5 \%)$ for LMI in view of the fact that only two of all cases of SMI exhibited ÂT lower than 195 degrees (Figure 2E). However, very modest sensitivity $(62.2 \%)$ impairs a value of $\hat{A} T$ of less than 195 degrees as a reliable index of LMI. The AUC for ÂT attained the highest values in discriminating
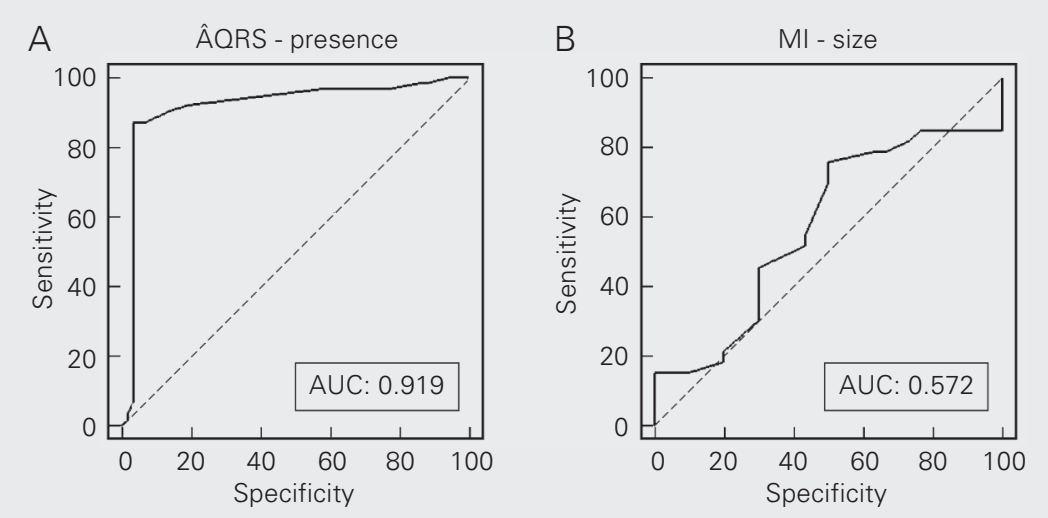

Figure 3. Use of receiver operating characteristic curves for ÂORS to identify the presence of myocardial infarction (panel $\mathrm{A}$ : presence of $\mathrm{MI}$ ) and to distinguish $\mathrm{MI}$ larger than $40 \%$ from infarction of less than $40 \%$ of left ventricular endocardial circumference (panel B: MI size). The values of the area under the curve (AUC) are also presented, as well as the line that indicates when the variable under study cannot distinguish between the two groups (AUC: 0.50; dashed line)
LMI from SMI among the indexes studied by us: 0.790 . When $\Sigma$ lower than $10 \mathrm{~mm}$ was tested for discriminating LMI from SMI a comparable AUC of 0.766 was found, corresponding to $33.3 \%$ sensitivity and $92.9 \%$ specificity (Figure 2F). Intriguingly, however, in our cases, animals showing $\Sigma<10$ $\mathrm{mm}$ were those with SMI instead of LMI. Indeed, only one of our SMI presented $\Sigma$ higher than $10 \mathrm{~mm}$ and $41 \%$ of the animals with LMI showed $\Sigma \geq 10 \mathrm{~mm}$. These results clearly exclude the sum of precordial $\mathrm{R}$ waves as a good index for LMI identification.

\section{Discussion}

In comparing our results with those reported by others, we faced a perplexing problem: inconceivable amplitudes for QRS waves are frequently reported by others. In fact, $\mathrm{Q}_{1}$ greater than $1 \mathrm{mV}$ and a sum of QRS positivity in precordial leads higher than 10 $\mathrm{mV}$ have been described (2-9). When using $2 \mathrm{~N}$ standardization for the ECG record, as done in the present study, these values will correspond to 20 and $200 \mathrm{~mm}$ in height for records of $\mathrm{Q}_{1}$ and $\mathrm{R}$ waves, respectively. Reported data are certainly puzzling, since these heights are incompatible with ECG records, making the reported data certainly equivocal. We prefer to report our values as $\mathrm{mm}$ in height. For comparison purposes, one must take into account the standardization used. In view of the animal facilities of the university, we preferred to utilize female rats in this study. Since there is no report of gender differences regarding the ECG pattern of MI, this does not seem to be a limitation of our results. In addition, as previously stated, the echocardiographic evaluation used here has proved to have an excellent correspondence with histological determinations of MI size, as assessed by linear correlation and by the Altman-Bland test (1).

Many reports consider a larger $\mathrm{Q}_{1}$ associated with a low $\Sigma$ to be a good criterion for selecting rats with LMI. Indeed, such crite- 
rion has been reported to screen over $95 \%$ of rats with LMI $(7,8)$. Our data markedly differ from previously reported results. In fact, although $\mathrm{Q}_{1}>1 \mathrm{~mm}$ emerged as a good marker for the presence of $\mathrm{MI}$ in the rat, it was recorded equally in LMI and SMI animals, thus showing that a large $\mathrm{Q}_{1}$ is far from specific for LMI. In addition, curiously, in our cases, $\Sigma$ lower than $10 \mathrm{~mm}$ was more frequently encountered in SMI than in LMI, showing that the height of precordial $\mathrm{R}$ waves does not discriminate LMI.

Our data permit us to conclude that routine nine-lead ECG permits the identification in an easy and quite reliable manner, the presence of $\mathrm{MI}$ in rats when a $\mathrm{Q}$ wave is present in $\mathrm{D}_{1}$, together with $\hat{\mathrm{A} Q R S}$ and $\hat{\mathrm{AT}}$ located between 0 and 240 degrees and $\Sigma$ lower than $10 \mathrm{~mm}$. Nevertheless, as is the case for routine clinical use of twelve leads in electrocardiographic studies of MI in humans, MI size cannot be characterized by previously described indexes. The well-accepted criterion based on deep $\mathrm{Q}_{1}$ and low amplitude of precordial $\mathrm{R}$ waves does not select larger than $40 \% \mathrm{MI}$.

\section{Acknowledgments}

Special thanks are due to Dr. Regina H.E. Alfarano, New York University and University of São Paulo, Brazil, for correcting the language and style of the manuscript.

\section{References}

1. Moisés VA, Ferreira RL, Nozawa E, Kanashiro RM, Campos Filho O, Andrade JL, Carvalho ACC \& Tucci PJF (2000). Structural and functional characteristics of rat hearts with and without myocardial infarct. Initial experience with Doppler echocardiography. Arquivos Brasileiros de Cardiologia, 75: 131-136.

2. Muller JE, Maroko P \& Braunwald E (1978). Praecordial electrocardiographic mapping. A technique to assess the efficacy of interventions designed to limit infarct size. Circulation, 57: 1-18.

3. Yusuf S, Lopez R, Maddison A, Maw PMS \& Sleight P (1979). Value of electrocardiogram in predicting and estimating infarct size in man. British Heart Journal, 42: 286-293.

4. Gay RG, Graham S, Aguirre M, Goldman S \& Morkin E (1988). Effects of 10- to 12-day treatment with L-thyroxine in rats with myocardial infarction. American Journal of Physiology, 24: H801H806.

5. Pfeffer MA, Pfeffer JM, Steinberg C \& Finn P (1985). Survival after an experimental myocardial infarction: beneficial effects of long- term therapy with captopril. Circulation, 72: 406-412.

6. Raya TE, Gay RG, Lancaster L, Aguirre M, Moffett C \& Goldman S (1988). Serial changes in left ventricular relaxation and chamber stiffness after large myocardial infarction in rats. Circulation, 77: 1424-1431.

7. Raya TE, Fonken SJ, Lee RW, Daugherty S, Goldman S, Wong PC, Timmermans PB \& Morkin E (1991). Hemodynamic effects of direct angiotensin II blockade compared to converting enzyme inhibition in a rat model of heart failure. American Journal of Hypertension, 4: 334S-340S.

8. Raya TE, Gay RG, Aguirre M \& Goldman S (1989). Importance of venodilation in prevention of left ventricular dilatation after chronic large myocardial infarction in rats: a comparison of captopril and hydralazine. Circulation Research, 64: 330-337.

9. Yang R, Bunting S, Gillet N, Clark R \& Jin H (1995). Congestive heart failure: growth hormone improves cardiac performance in experimental heart failure. Circulation, 92: 262-267. 